Supporting Information

\title{
Extraordinarily Persistent Zero Linear Compressibility in Metal-organic Framework MIL-122(In)
}

Yishuai $\mathrm{Yu}^{\dagger}$, Qingxin Zeng ${ }^{\ddagger}$, Yaping Chen ${ }^{\dagger}$, Lina Jiang ${ }^{\dagger}$, Kai Wang ${ }^{\dagger} *$, and Bo Zou ${ }^{\dagger} *$

${ }^{\dagger}$ State Key Laboratory of Superhard Materials, College of Physics, Jilin University,

Changchun, 130012, China

${ }^{\ddagger}$ Laboratory of Quantum Materials Design and Application, School of Physics and Electronic Engineering, Jiangsu Normal University, Xuzhou 221116, China 


\section{Sample Preparation.}

MIL-122(In) was synthesized using the reported procedure. ${ }^{[1]}$ The compound $\mathrm{In}_{2}(\mathrm{OH})_{2}\left[\mathrm{C}_{14} \mathrm{H}_{4} \mathrm{O}_{8}\right]$, MIL-122(In) have been hydrothermally synthesized under autogenous pressure. The starting mixture were $\mathrm{In}\left(\mathrm{NO}_{3}\right)_{3} \cdot \mathrm{xH}_{2} \mathrm{O}(0.339 \mathrm{~g}, 0.9 \mathrm{mmol}$, Aladdin $99.999 \%)$, 1,4,5,8-naphthalenetetracarboxylic acid $\left(\mathrm{C}_{14} \mathrm{H}_{8} \mathrm{O}_{8}, 0.135 \mathrm{~g}, 0.4 \mathrm{mmol}\right.$, TCI 95\%) and $\mathrm{H}_{2} \mathrm{O}(5 \mathrm{ml}$, $277.8 \mathrm{mmol})$. The mixtures of the different reactants have been placed in a $50 \mathrm{ml}$ Teflon-lined stainless-steel autoclave and heated for $24 \mathrm{~h}$ at $210{ }^{\circ} \mathrm{C}$. After standing time for cooling, the powdered compound was washed with N,N-dimethylformamide (DMF) and dried in vacuum for $12 \mathrm{~h}$.

\section{High-pressure generation.}

High-pressure experiment were carried out by a symmetric diamond anvil cell (DAC). A T301 steel gasket was pre-indented to a thickness of $40 \mu \mathrm{m}$. A $0.15 \mathrm{~mm}$ sized cavity was drilled in the gasket for loading the samples. A ruby ball was used to gauge the pressure by the R1 ruby fluorescence method. Silicon oil was used as the pressure transmitting medium.

\section{ADXRD Measurements.}

In situ high-pressure ADXRD experiments was performed with a wavelength of $0.6199 \AA$ beam at beamline BL15U1, Shanghai Synchrotron Radiation Facility (SSRF), China. $\mathrm{CeO}_{2}$ was used as the standard sample for calibration. The Bragg diffraction rings were integrated to yield 1D intensity versus diffraction angle 2-theta patterns using Fit2D program. The lattice parameters were extracted from the ADXRD data using the program of Materials Studio by Pawley refinement. The compressibility was obtained using the empirical form:

$l=l_{0}+\lambda \cdot\left(p-p_{c}\right)^{v}$ by the free online program PASCal from http://pascal.chem.ox.ac.uk/. ${ }^{[2]}$

\section{Computational Methodology.}

$\mathrm{Ab}$ initio calculations were performed with the pseudopotential plane-wave methods based on density functional theory implemented in the CASTEP package at the GGA-DFT level with the PBE exchange correlation functional. PBE-D2 is applied as dispersion corrections. The $1 \times$ $1 \times 1$ unit cell of the reported ambient structure of MIL-122(In) with 2 formula units in a unit cell was used as a structural model. The plane-wave cutoff energy and Monkhorst-Pack grid for the electronic Brillouin zone integration were $340 \mathrm{eV}$ and $1 \times 1 \times 2$, respectively. The selfconsistent field (SCF) tolerance was set as $1.0 \times 10^{-6} \mathrm{eV} /$ atom. The optimization is stopped when stress and forces on the atoms were less than $0.05 \mathrm{GPa}$ and $0.03 \mathrm{eV} / \AA$, respectively. 
The principal axes and lattice axes are related by the matrix algebra ${ }^{[2]}$ :

$$
\left[\begin{array}{l}
x_{1} \\
x_{2} \\
x_{3}
\end{array}\right]=\left[\begin{array}{ccc}
0 & 1 & 0 \\
0.0388 & 0 & 0.9993 \\
0.9999 & 0 & -0.0126
\end{array}\right]\left[\begin{array}{l}
a \\
b \\
c
\end{array}\right]
$$

The deviation of $\mathrm{X}_{2}$ and $\mathrm{X}_{3}$ away from the $a$ and $c$-axes is less than $1 \%$, so we use the lattice axis to describe the linear compressibility.

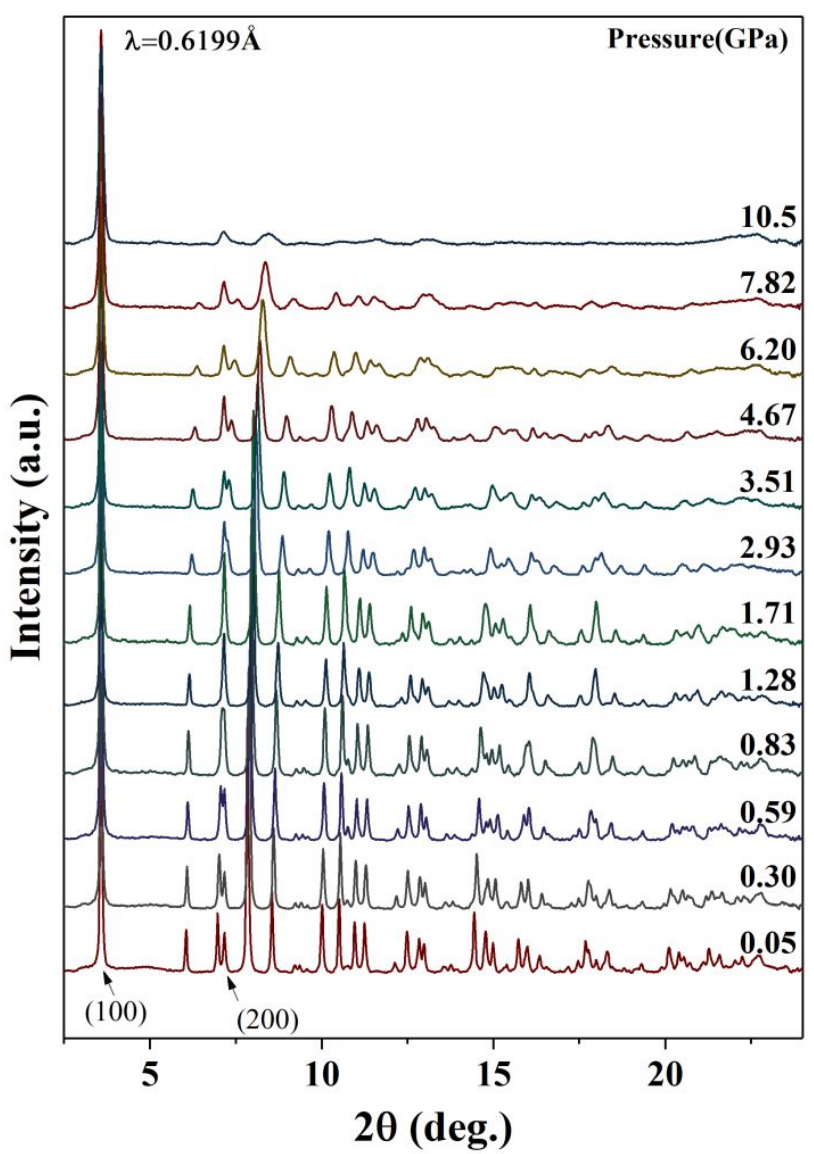

Figure S1. The evolution of represent ADXRD patterns of MIL-122(In) under compression. 


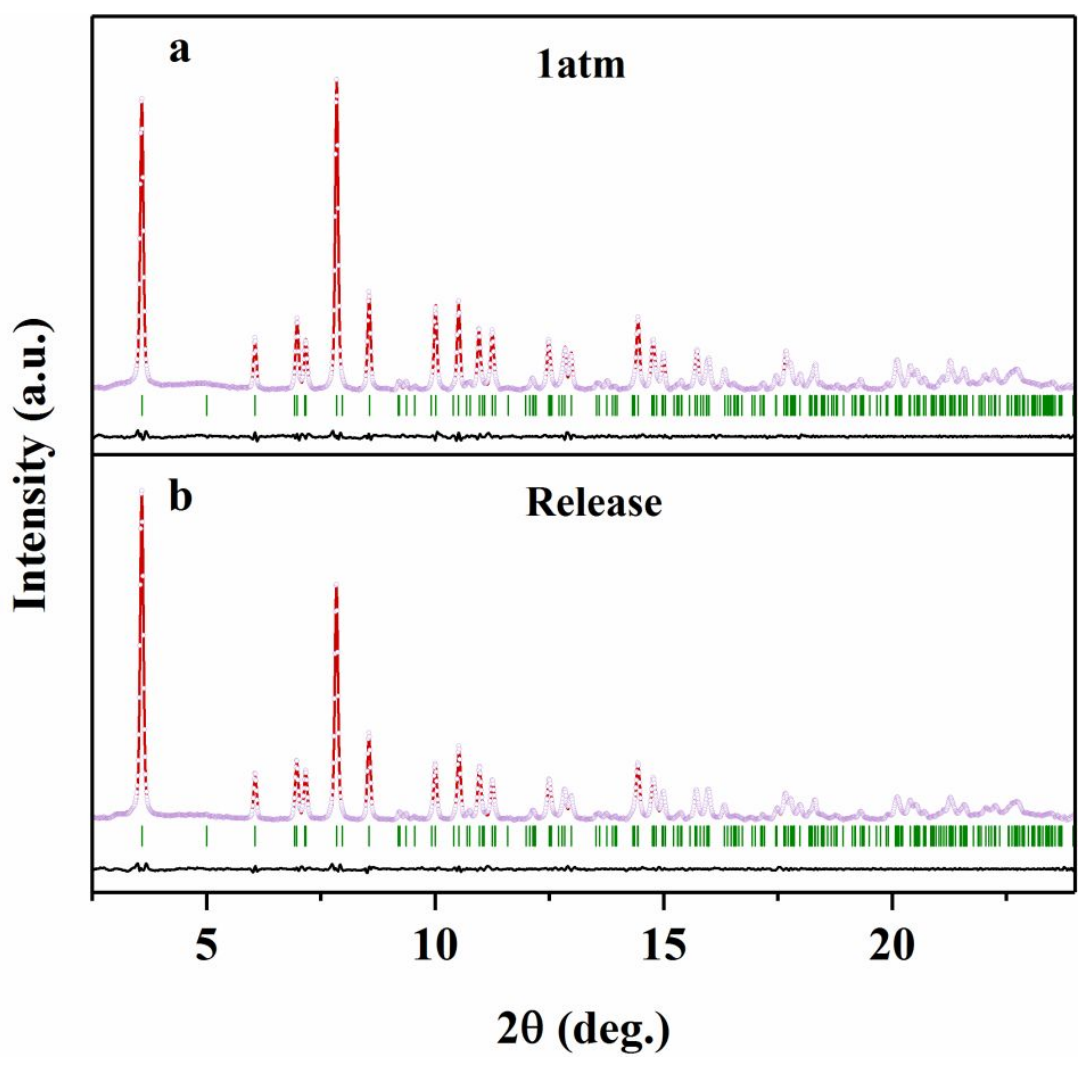

Figure S2. Refinement of the ADXRD pattern of MIL-122(In) at (a) ambient condition and (b) decompression, respectively. Green line reflect the refined peak positions and the black line represents the differences between observed (points) and simulated (solid line) profiles.

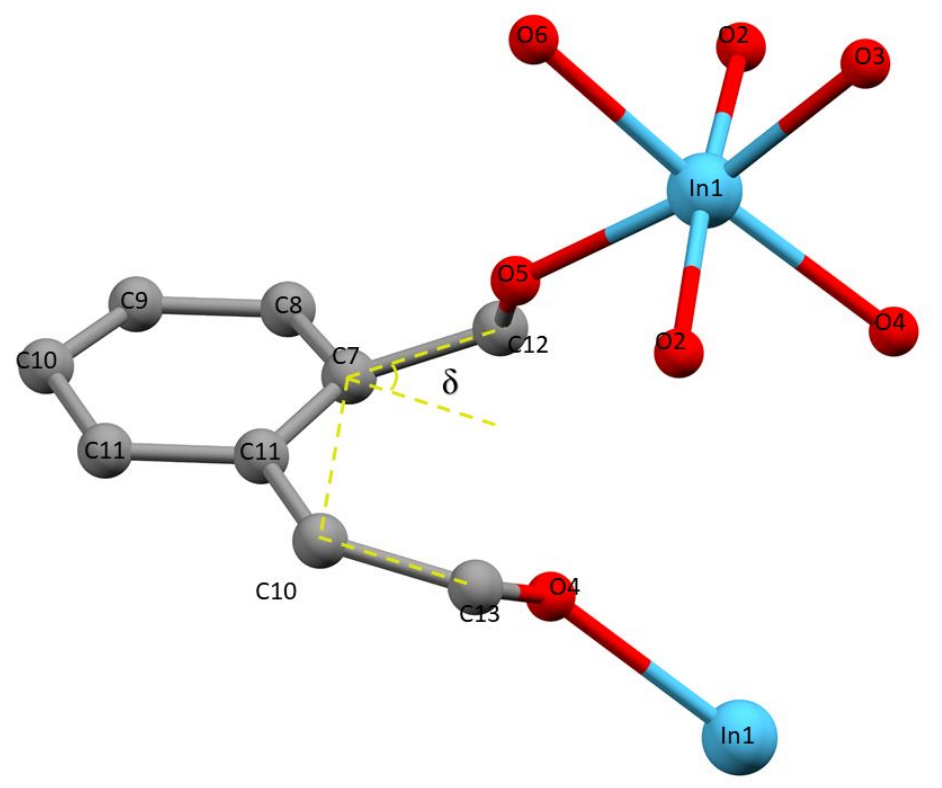

Figure S3. The X-ray structure of the torsion angle $\delta(\mathrm{C} 12-\mathrm{C} 7-\mathrm{C} 10-\mathrm{C} 13)$. 

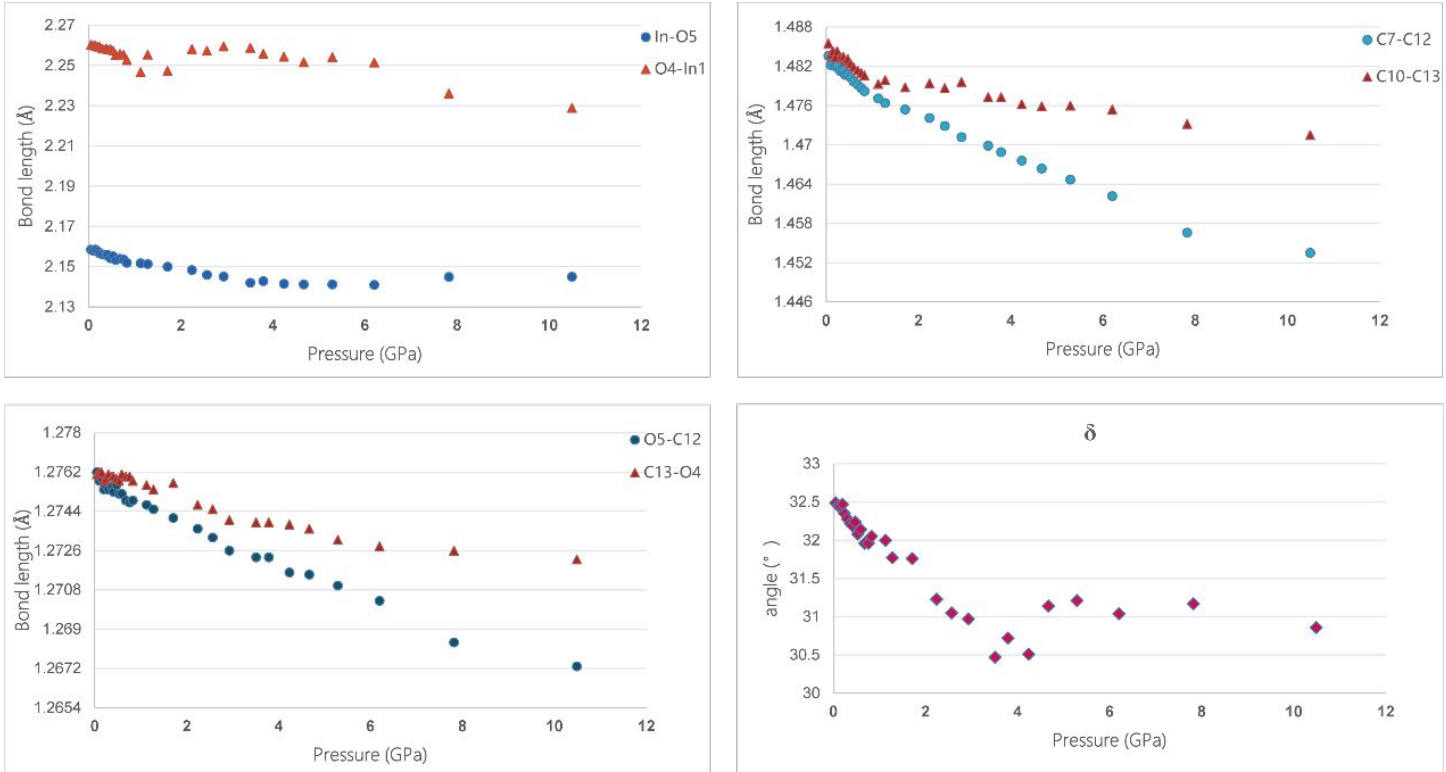

Figure S4. The evolution of the key bond length and angle in XBU.

We replaced the In element with the element Ga to verified that MIL-122(Ga) with the same topology structure of MIL-122(In) also has zero linear compressibility.
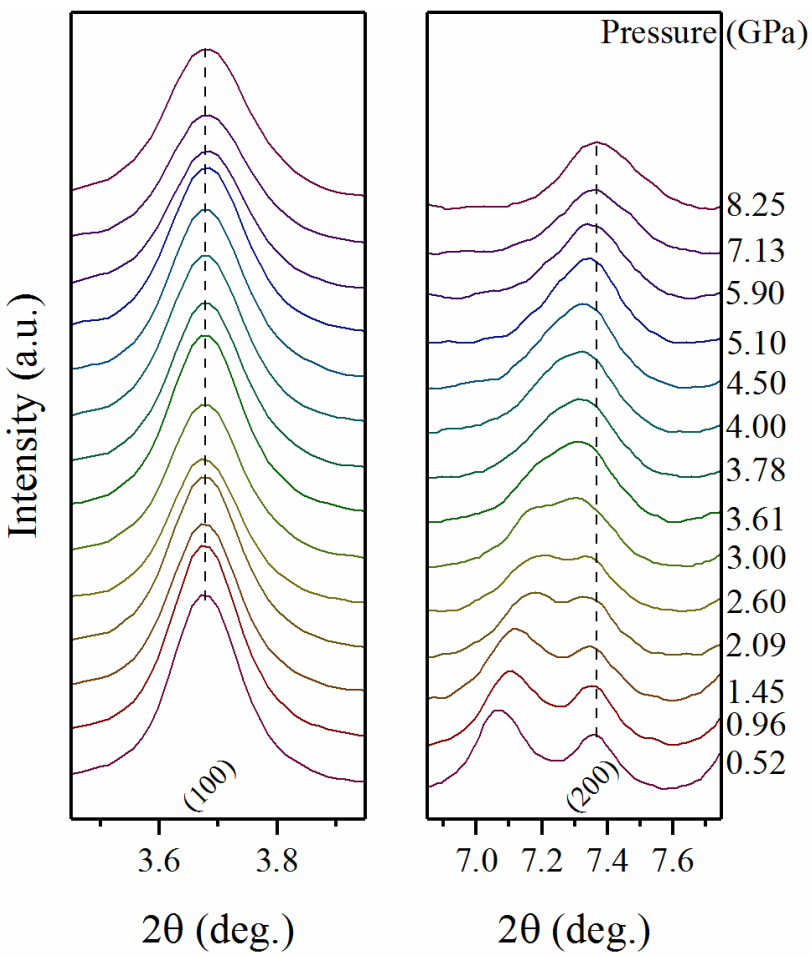

Figure S5.: ADXRD spectra of MIL-122(Ga) at various pressures. As the same as MIL122(In), the Bragg peak of (100) of MIL-122(Ga) didn't shift toward high-angle during the course of pressurization. 
Table S1. Lattice parameters data by geometry optimization calculations.

\begin{tabular}{|c|c|c|c|c|c|}
\hline $\mathrm{P}(\mathrm{GPa})$ & a $(\AA)$ & $\mathrm{b}(\AA)$ & c $(\AA)$ & $\beta\left(^{\circ}\right)$ & $\alpha=\gamma\left({ }^{\circ}\right)$ \\
\hline $1 \mathrm{~atm}$ & 9.9236 & 10.1946 & 7.1935 & 92.5503 & 90 \\
\hline 0.05 & 9.9259 & 10.1838 & 7.1827 & 92.5538 & 90 \\
\hline 0.15 & 9.9262 & 10.1675 & 7.1772 & 92.5552 & 90 \\
\hline 0.3 & 9.9265 & 10.1178 & 7.1605 & 92.6024 & 90 \\
\hline 0.37 & 9.9268 & 10.1055 & 7.1563 & 92.6037 & 90 \\
\hline 0.41 & 9.9271 & 10.0963 & 7.1530 & 92.6091 & 90 \\
\hline 0.47 & 9.9269 & 10.0848 & 7.1489 & 92.6098 & 90 \\
\hline 0.59 & 9.9275 & 10.0579 & 7.1402 & 92.6240 & 90 \\
\hline 0.68 & 9.9292 & 10.0421 & 7.1344 & 92.6383 & 90 \\
\hline 0.83 & 9.9287 & 10.0185 & 7.1217 & 92.6408 & 90 \\
\hline 0.96 & 9.9299 & 9.9957 & 7.1113 & 92.6531 & 90 \\
\hline 1.13 & 9.9315 & 9.9695 & 7.1026 & 92.6426 & 90 \\
\hline 1.28 & 9.9329 & 9.9540 & 7.0972 & 92.6401 & 90 \\
\hline 1.71 & 9.9335 & 9.9127 & 7.0859 & 92.6282 & 90 \\
\hline 1.97 & 9.9350 & 9.8832 & 7.0746 & 92.6128 & 90 \\
\hline 2.24 & 9.9372 & 9.8563 & 7.0662 & 92.6603 & 90 \\
\hline 2.57 & 9.9383 & 9.8140 & 7.0510 & 92.6958 & 90 \\
\hline 2.93 & 9.9387 & 9.7747 & 7.0278 & 92.6556 & 90 \\
\hline 3.2 & 9.9405 & 9.7494 & 7.0174 & 92.6842 & 90 \\
\hline 3.51 & 9.9414 & 9.7178 & 7.0017 & 92.6994 & 90 \\
\hline 3.79 & 9.9399 & 9.6954 & 6.9936 & 92.6448 & 90 \\
\hline 4.24 & 9.9392 & 9.6633 & 6.9718 & 92.5457 & 90 \\
\hline 4.67 & 9.9389 & 9.6205 & 6.9561 & 92.4972 & 90 \\
\hline 5.29 & 9.9370 & 9.5759 & 6.9340 & 92.3632 & 90 \\
\hline 6.2 & 9.9370 & 9.5042 & 6.9069 & 92.2536 & 90 \\
\hline 7.82 & 9.9253 & 9.4054 & 6.8369 & 91.5098 & 90 \\
\hline 10.5 & 9.9238 & 9.2849 & 6.8264 & 91.3184 & 90 \\
\hline
\end{tabular}


Table S2. The data of lattice parameters in the MIL-122(In) and the XBU unit.

\begin{tabular}{|c|c|c|c|c|}
\hline $\mathrm{P}(\mathrm{GPa})$ & a $(\AA)$ & $\mathrm{a}^{\prime}-\mathrm{XBU}(\AA)$ & Error(\%o) & $\Delta \mathrm{a} \%$ \\
\hline $1 \mathrm{~atm}$ & $9.9236(1)$ & $9.9234(9)$ & 0.013 & 0 \\
\hline 0.05 & $9.9259(2)$ & $9.9260(8)$ & 0.015 & 0.023 \\
\hline 0.15 & $9.9262(2)$ & $9.9259(0)$ & 0.032 & 0.026 \\
\hline 0.3 & $9.9265(9)$ & $9.9266(8)$ & 0.009 & 0.030 \\
\hline 0.37 & $9.9268(0)$ & $9.9268(8)$ & 0.009 & 0.032 \\
\hline 0.41 & $9.9271(9)$ & $9.9270(1)$ & 0.018 & 0.036 \\
\hline 0.47 & $9.9269(3)$ & $9.9268(7)$ & 0.007 & 0.033 \\
\hline 0.59 & $9.9275(1)$ & $9.9268(3)$ & 0.068 & 0.039 \\
\hline 0.68 & $9.9292(8)$ & $9.9287(1)$ & 0.057 & 0.057 \\
\hline 0.83 & $9.9287(1)$ & $9.9283(5)$ & 0.037 & 0.051 \\
\hline 0.96 & $9.9299(8)$ & $9.9294(1)$ & 0.057 & 0.064 \\
\hline 1.13 & $9.9315(2)$ & $9.9313(1)$ & 0.021 & 0.080 \\
\hline 1.28 & $9.9329(6)$ & $9.9325(1)$ & 0.045 & 0.094 \\
\hline 1.71 & $9.9335(4)$ & $9.9326(6)$ & 0.089 & 0.100 \\
\hline 1.97 & $9.9350(2)$ & $9.9351(7)$ & 0.015 & 0.115 \\
\hline 2.24 & $9.9372(4)$ & $9.9364(9)$ & 0.076 & 0.137 \\
\hline 2.57 & $9.9383(5)$ & $9.9378(0)$ & 0.055 & 0.149 \\
\hline 2.93 & $9.9387(6)$ & $9.9380(8)$ & 0.068 & 0.153 \\
\hline 3.2 & $9.9405(8)$ & $9.9411(4)$ & 0.056 & 0.171 \\
\hline 3.51 & $9.9414(4)$ & $9.9407(2)$ & 0.072 & 0.180 \\
\hline 3.79 & $9.9399(8)$ & $9.9400(5)$ & 0.007 & 0.165 \\
\hline 4.24 & $9.9392(3)$ & 9.9384(4) & 0.080 & 0.157 \\
\hline 4.67 & $9.9389(6)$ & $9.9391(3)$ & 0.017 & 0.155 \\
\hline 5.29 & $9.9370(8)$ & $9.9369(9)$ & 0.008 & 0.136 \\
\hline 6.2 & $9.9370(0)$ & $9.9365(2)$ & 0.048 & 0.135 \\
\hline 7.82 & $9.9253(2)$ & $9.9243(9)$ & 0.093 & 0.017 \\
\hline 10.5 & $9.9238(3)$ & $9.9229(8)$ & 0.086 & 0.002 \\
\hline
\end{tabular}


Table S3. The cell parameters extracted from the high-pressure XRD patterns.

\begin{tabular}{|c|c|c|c|c|c|}
\hline $\mathrm{P}(\mathrm{GPa})$ & $\mathrm{a}(\AA)$ & $\mathrm{b}(\AA)$ & $\mathrm{c}(\AA)$ & $\beta\left(^{\circ}\right)$ & $\alpha=\gamma\left({ }^{\circ}\right)$ \\
\hline $1 \mathrm{~atm}$ & 9.9236 & 10.1946 & 7.1935 & 92.5503 & 90 \\
\hline 0.05 & 9.9259 & 10.1838 & 7.1827 & 92.5538 & 90 \\
\hline 0.15 & 9.9247 & 10.1622 & 7.1753 & 92.5837 & 90 \\
\hline 0.3 & 9.9262 & 10.1675 & 7.1771 & 92.5552 & 90 \\
\hline 0.37 & 9.9251 & 10.1411 & 7.1681 & 92.5938 & 90 \\
\hline 0.41 & 9.9261 & 10.1323 & 7.1652 & 92.5975 & 90 \\
\hline 0.47 & 9.9265 & 10.1178 & 7.1605 & 92.6024 & 90 \\
\hline 0.59 & 9.9267 & 10.1055 & 7.1563 & 92.6037 & 90 \\
\hline 0.68 & 9.9271 & 10.0963 & 7.1530 & 92.6091 & 90 \\
\hline 0.83 & 9.9269 & 10.0848 & 7.1489 & 92.6098 & 90 \\
\hline 0.96 & 9.9280 & 10.0738 & 7.1450 & 92.6215 & 90 \\
\hline 1.13 & 9.9275 & 10.0578 & 7.1402 & 92.6240 & 90 \\
\hline 1.28 & 9.9292 & 10.0421 & 7.1344 & 92.6383 & 90 \\
\hline 1.71 & 9.9291 & 10.0312 & 7.1280 & 92.6490 & 90 \\
\hline 1.97 & 9.9287 & 10.0184 & 7.1217 & 92.6408 & 90 \\
\hline 2.24 & 9.9299 & 9.9957 & 7.1113 & 92.6531 & 90 \\
\hline 2.57 & 9.9315 & 9.9695 & 7.1026 & 92.6426 & 90 \\
\hline 2.93 & 9.9329 & 9.9540 & 7.0972 & 92.6401 & 90 \\
\hline 3.2 & 9.9328 & 9.9402 & 7.0943 & 92.6172 & 90 \\
\hline 3.51 & 9.9335 & 9.9127 & 7.0859 & 92.6282 & 90 \\
\hline 3.79 & 9.9350 & 9.8832 & 7.0746 & 92.6128 & 90 \\
\hline 4.24 & 9.9372 & 9.8563 & 7.0661 & 92.6603 & 90 \\
\hline 4.67 & 9.9383 & 9.8140 & 7.0509 & 92.6958 & 90 \\
\hline 5.29 & 9.9387 & 9.7747 & 7.0278 & 92.6556 & 90 \\
\hline 6.2 & 9.9405 & 9.7494 & 7.0174 & 92.6842 & 90 \\
\hline 7.82 & 9.9414 & 9.7178 & 7.0017 & 92.6994 & 90 \\
\hline 10.5 & 9.9399 & 9.6954 & 6.9936 & 92.6448 & 90 \\
\hline
\end{tabular}

\section{REFERENCES}

[1] Volkringer C, Loiseau T, Guillou N, Férey G, Elkaïm E. Solid State Sciences. 2009;11(8):1507-12.

[2] Cliffe, M. J.; Goodwin, A. L. J. Appl. Crystallogr. 2012, 45, 1321-1329. 